\title{
INFLUÊNCIA DA DOR CRÔNICA NA CAPACIDADE FUNCIONAL, FRAGILIDADE E EM CONDIÇÕES DE SAÚDE DE IDOSOS
}

Areta Dames Cachapuz Novaes; Universidade Federal de São Carlos; aretanovaes@estudante.ufscar.br; Loren Bettoni Blanco; Universidade Federal de São Carlos; Ioren.bett@hotmail.com; José Emanuel Alves; Universidade Federal de São Carlos; joseemanuelalves@estudante.ufscar.br; Karina Gramani-Say; Universidade Federal de São Carlos; gramanisay@ufscar.br

\section{RESUMO}

Introdução: No envelhecimento a prevalência pode ser alta de doenças crônicas e degenerativas, de dor crônica e dependência para a realização de atividades de vida diária e redução da mobilidade. A dor crônica está entre a condição crônica mais prevalente encontrada associada a quedas. Objetivo: Verificar a influência da dor crônica na capacidade funcional, no equilíbrio postural, na fragilidade, e outras condições de saúde dos idosos. Metodologia: Corte transversal (CEP3332310) em idosos acima dos 60 anos com dor crônica há pelo menos 6 meses, da Oficina de Prevenção de Quedas na Atenção Básica. Divididos em grupos: caidor (GDC) $(n=11)$ pelo menos duas quedas nos últimos doze meses e não caidor (GDNC) $(n=12)$ sem relato de quedas. Foi avaliado o tempo, intensidade e local da dor, Atividades de Vida Diária, cognição, desempenho funcional e fenótipo de Fried. Foi realizado o teste T-Student e Mann-Whitney ( $\mathrm{p} \leq 0,05$; STATISTICA 7.0) Resultados: A intensidade média de dor relatada foi de 5,95 $( \pm 2,07), 12$ idosos relataram dor em 1 local do corpo (53\%), enquanto 11 relataram em 2 locais ou mais (43\%), com duração média de $121,04( \pm 121,84)$ meses, não houve diferença entre os grupos para a variável dor. Evidenciou-se diferença significativa para a variável fragilidade $(\mathrm{p}=0,03)$ para o GDNC em relação ao GDC. Não houve diferença para as demais variáveis avaliadas. Conclusão: Especificamente nesse grupo de Prevenção de Quedas, a dor crônica associada ao autorrelato de quedas não influenciou em perdas funcionais e/ou piores condições de saúde.

Palavras-chave: Envelhecimento; Dor crônica; Acidentes por quedas; Equilíbrio postural; Fragilidade. 